\title{
Angiostrongylus costaricensis and Experimental Infection of Sarasinula marginata - II. Elimination Routes
}

\author{
Cristiane Lafeta Gomes Furtado Mendonça, Omar Santos Carvalho, Ester Maria Mota*, \\ Marcelo Pelajo-Machado*, Luzia Fátima Gonçalves Caputo*, Henrique Leonel Lenzi*/+
} Centro de Pesquisas René Rachou-Fiocruz, Belo Horizonte, MG, Brasil *Departamento de Patologia, Instituto Oswaldo Cruz-
Fiocruz, Av. Brasil 4365, 21045-900 Rio de Janeiro, RJ, Brasil

Angiostrongylus costaricensis intermediate hosts are terrestrial mollusks mostly belonging to the Veronicellidae family. In the present investigation we focused on the mechanisms of larval expulsion from Sarasinula marginata infected with A. costaricensis. Twenty-five mollusks were individually infected with $5000 L_{1}$ and sacrificed at $30 \mathrm{~min}$ and 1, 2, 4, 6, and 8 host-infection and at days 1, 2, 4, 5, 6, 8, 10, 11, 12, 14, 15, 16, 20, 21, 22, 25, 26, 28, and 30 post-infection; the mollusks were then fixed and stained. Diverse organs involved throughout the course of the migratory routes of larvae from oral penetration on were specified and the mechanisms of larval access to the fibromuscular layer through the kidney, rectum, and vascular system were defined. The elimination of $L_{3}$, derived from oral and/or cutaneous infections, appears to depend on granulomas located close to the excretory ducts of mucous cells.

Key words: Angiostrongylus costaricensis - Sarasinula marginata - Veronicellidae - migratory routes - larval elimination amebocyte - invertebrate granuloma

Angiostrongylus costaricensis (Morera \& Céspedes 1971a) is the aetiological agent of abdominal angiostrongyliasis. Its life cycle in invertebrate hosts begins with ingestion of the larval first stage $\left(\mathrm{L}_{1}\right)$ by the mollusk and/or percutaneous penetration by the larva, followed by two moultings $\left(\mathrm{L}_{1} / \mathrm{L}_{2} / \mathrm{L}_{3}\right)$ in the fibromuscular layer and, exceptionally, inside the viscera. Larvae $\mathrm{L}_{3}$ are eliminated together with the mucous secretion of the mollusk (Morera \& Céspedes 1971b, Conejo \& Morera 1988, Thiengo 1996).

In our previous work, studying the life cycle of $A$. costaricensis in Sarasinula marginata (Semper, 1888), we confirmed the simultaneous occurrence of oral and percutaneous infection of mollusks and described in detail the mode and sites of $\mathrm{L}_{1}$ penetration into the mollusk. The involvement of several organs during the migration of the parasite in the host tissues was also reported and the cellular perilarval (pre-granuloma and granuloma) and systemic (hemocytosis) reactions in the mollusk were characterized (Mendonça et al. 1999). It is important to stress that this mollusk has never been found naturally infected.

Here we focus on the probable mechanism, which promotes larval elimination together with the mollusk's mucous secretion, and we also add more details to the previous report better clarifying the migratory routes of A. costaricensis in the intermediate host.

Partial financial support: Papes 383-Fiocruz

${ }^{+}$Corresponding author. Fax: +55-21-2573.8673. E-mail: hlenzi@ioc.fiocruz.br

Received 17 September 2002

Accepted 19 September 2003

\section{MATERIALS AND METHODS}

Twenty-five $S$. marginata slugs captured in vegetable gardens in the city of Rio de Janeiro, where no evidence of natural infection was detected, weighing about $1 \mathrm{~g}$, were individually kept in plastic containers and fasted for 5 days. They were then exposed to larvae $\mathrm{L}_{1}$ of $A$. costaricensis (Criciumal strain) by feeding on circular lettuce fragments $(1 \mathrm{~cm}$ in diameter) covered with approximately 5000 larvae concentrated in $100 \mu$ of a liquid solution of healthy mouse feces. The slugs were killed at $30 \mathrm{~min}, 1,2,4,6,8 \mathrm{~h}$, and at $1,2,4,5,6,8,10,11,12$, $14,15,16,20,21,22,25,26,28,30$ days after infection, and fixed in Carson's Formalin-Millonig (Carson et al. 1973). Serial cross-sections from mollusk's whole body were stained with Haematoxylin-Eosin (HE) and/or Lennert's Giemsa (Lennert 1978) and all slides (about 500 per slug) were examined by brightfield microscopy. For documentation, selected slides were analyzed with a Zeiss photomicroscope III and the images were captured with a chilled Hamamatsu color 3 CCD camera (model C-5810), stored in tagged-image file format (TIFF) and printed with a Codonics TM NP-1600 photographic dye-sublimation printer or Deskjet 930C (Hewlett Packard).

\section{RESULTS}

After $1 \mathrm{~h}, \mathrm{~L}_{1}$ were occasionally detected inside peripheral vessels of the subtegumental vascular network (Fig. 1) and $2 \mathrm{~h}$ after infection they were also found into the vessels with intermittent muscular sphincters, located in the fibromuscular layer (Fig. 2), and in some narrow vessels at the renal periphery. After $4 \mathrm{~h}$ of infection, $\mathrm{L}_{1}$ reduced in number within the digestive tract lumen, being also found in the fibromuscular layer, buccal bulb, esophagus, crop, stomach, digestive gland, and intestine. From $6 \mathrm{~h}$ of infection on, larvae were mainly located in the fibromuscular layer and were also found in large numbers in the peripheral and interstitial portions of the kidney (Fig. 3) and rectum 
(Fig. 4). Few of them were detected in the same organs as described above in addition to the ureters and vessels eventually in the sheath of the penis, ovariotestis, oviduct, copulation purse, coeloma (Fig. 5), and salivary, pedal and penial glands. Almost all larvae were surrounded by a pregranulomatous cellular reaction (Table).
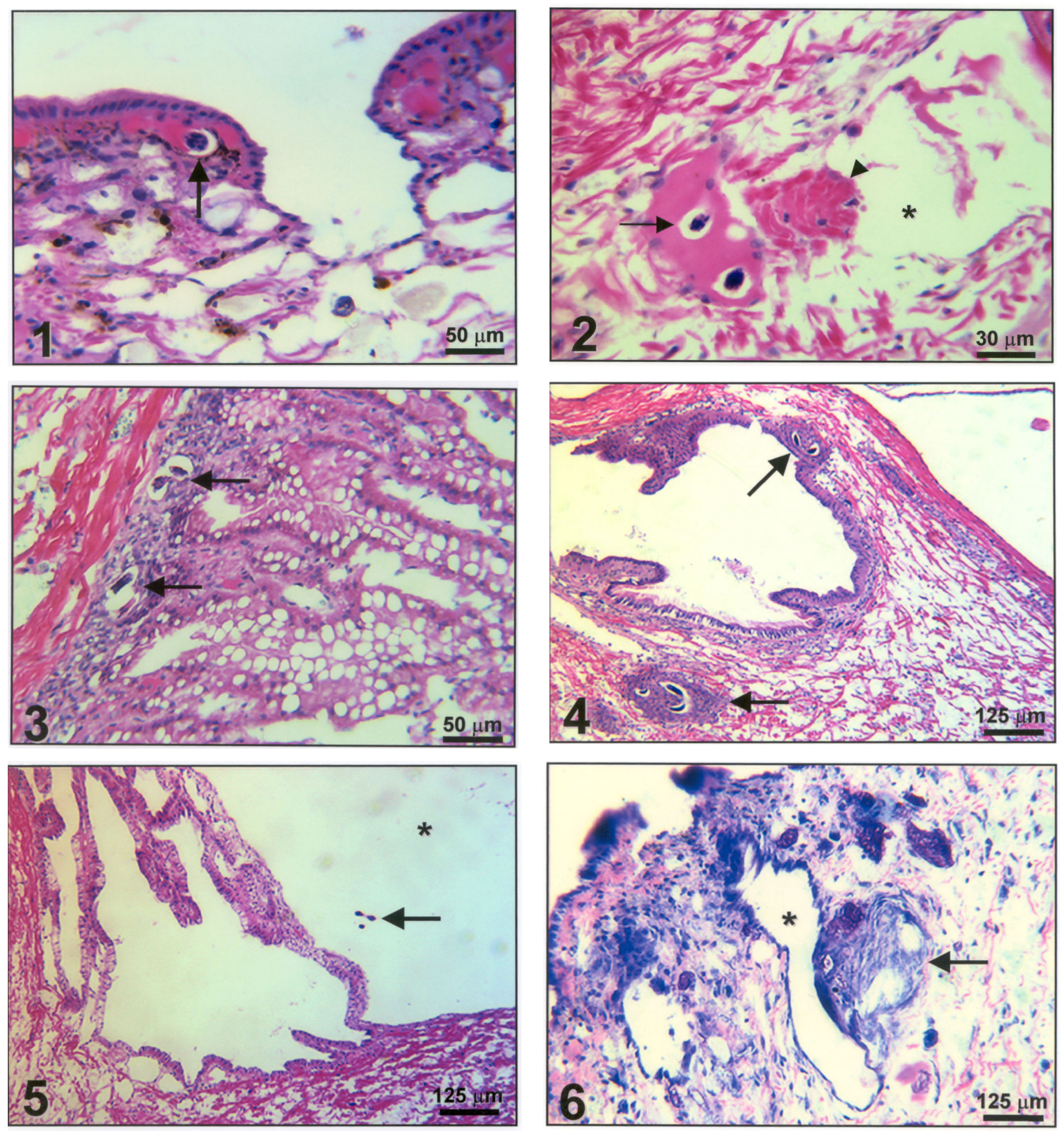

Fig. 1: larva inside a small vessel (arrow) in the subtegumental tissue (time of infection: $1 \mathrm{~h}$ ) (HE, 200x). Fig. 2: two vascular segments of one meta-artery-like vessel interposed by a muscular sphincter (arrow head), with one of them full of proteinaceous eosinophilic material around larvae (arrow), while the other is empty (asterisk) (time of infection: $2 \mathrm{~h}$ ) (HE, 310x). Fig. 3: larvae $\left(\mathrm{L}_{1}\right)$ in perirenal vessels (arrows) surrounded by a slight amebocyte reaction (time of infection: $24 \mathrm{~h}$ ) (HE, 200x). Fig. 4: granulomas in the fibromuscular tissue contiguous to the rectum. The granuloma centered at the top of the figure (indicated by an oblique arrow) is located at the side of the hemocoelome cavity (upper right corner), while the other one (horizontal arrow) is placed in the fibromuscular layer adjacent to the epidermis (not shown in the figure) (time of infection: 4 days) (HE, 80x). Fig. 5: larvae inside the coelome (asterisk) near the kidney (arrow) (time of infection: $6 \mathrm{~h}$ ) (HE, 80x). Fig. 6: granuloma containing larvae (arrow) compressing the lumen of an excretory duct (asterisk) (time of infection: 4 days) (Giemsa, 80x) 


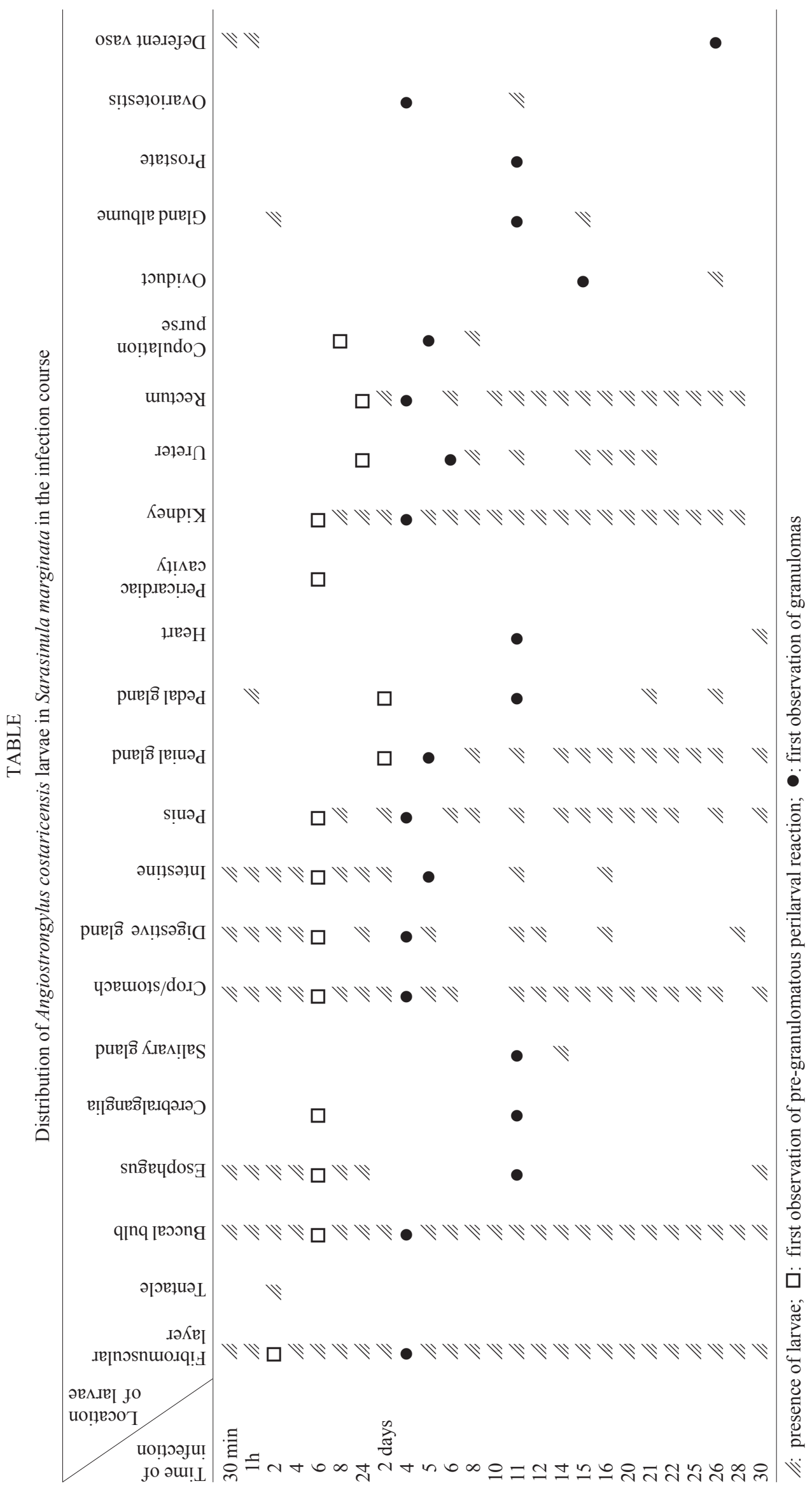


vessels of the fibromuscular layer. This aspect predominated from the 21th day of infection on. Some of the granulomas were positioned near the excretory ducts of the mucous cells (Figs 6, 7, 8). After 26 days of infection, the granulomas were mainly located intravascularly, adhering strongly to the endothelium through very thin septa (Figs 9,10) creating a sort of "peripheral or marginal sinus" between their core and the endothelial covering. Partial obstruction of the vascular lumen by the granulomas caused a diffuse thickness of vascular walls (Fig. 11) and dilation of meta-arterial-like vessels (Fig. 12). These vessels were characterized by the intermittent alternation of muscular sphincters and segments with an expanded lumen (manuscript in preparation).
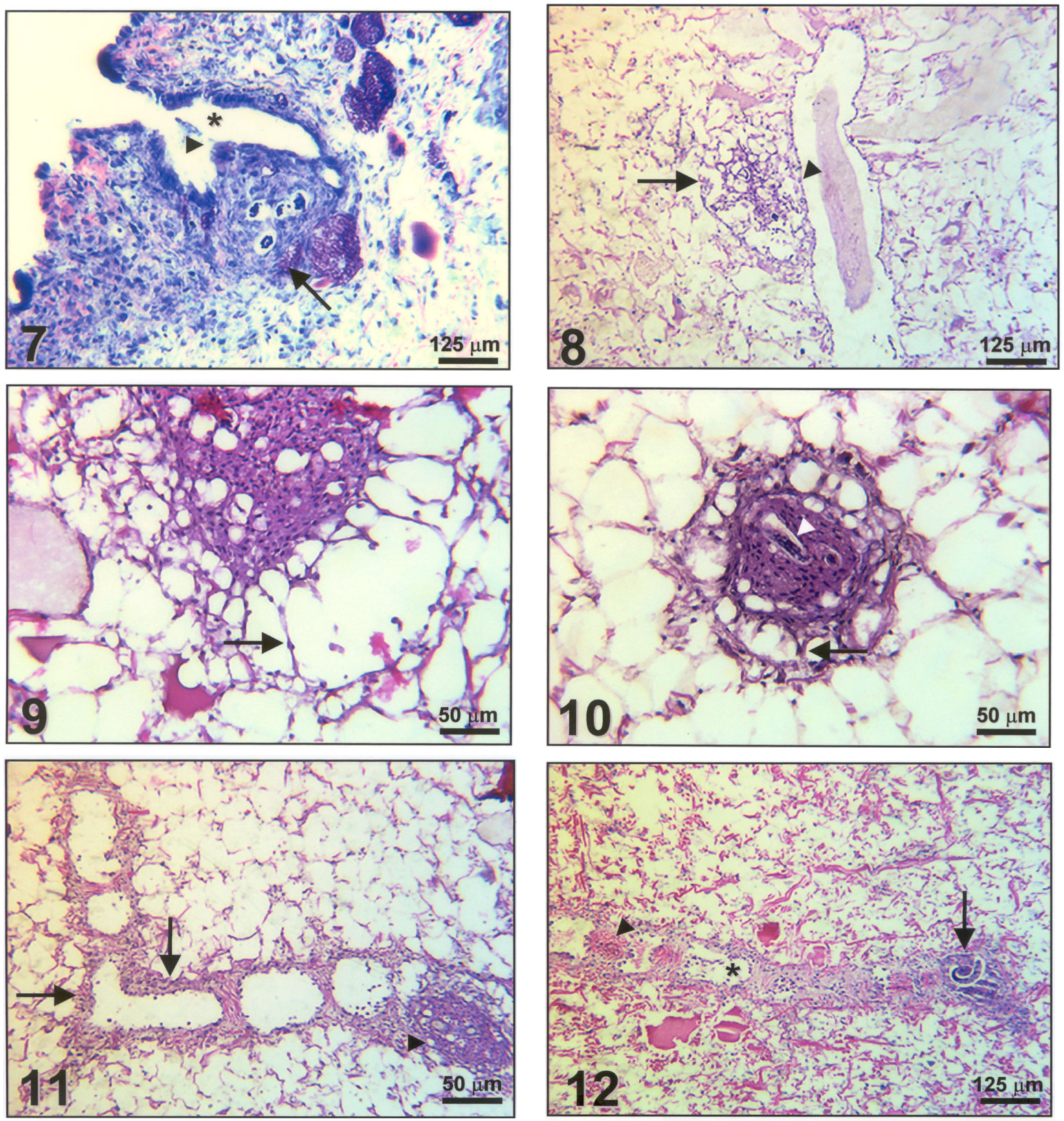

Fig. 7: granuloma (arrow) located at the botton of one excretory duct (asterisk) provoking partial rupture of its epithelial lining (arrow head) (time of infection: 4 days) (Giemsa, 80x). Fig. 8: intravascular granuloma (arrow) touching the epithelium of one duct (arrow head) (time of infection: 21 days) (HE, 80x). Fig. 9: intravascular granuloma showing an amebocyte-amebocyte network (inter-amebocytic) at the periphery, connected to the endothelium by long and fine septa (amebocyte-endothelium network) (time of infection: 30 days (HE, 200x). Fig. 10: intravascular granuloma with a "marginal sinus" rich in septa (arrow) centered by larvae (arrow head) (time of infection: 30 days) (HE, 200x). Fig. 11: granuloma (arrow head) adhered to the wall of one of the chambers of a meta-artery-like vessel, which presents diffuse thickning of its wall (arrow) (time of infection: 30 days) (HE, 200x). Fig. 12: larvae (arrow) inside a meta-artery-like vessel consisting of sphincters (arrow head) and segments with open lumen (asterisk) containing amebocytes (time of infection: 4 days) (HE, 80x) 
In Fig. 13, we propose a general and simplified scheme based on the present and previous results about penetration sites (Mendonça et al. 1999) and migration routes of $A$. costaricensis in $S$. marginata, considering also the possible mechanisms of larval release to the external environment.

\section{DISCUSSION}

The current work detailed the migratory routes of $A$. costaricensis in S. marginata and the putative mechanisms of $\mathrm{L}_{3}$ expulsion from the mollusk's body. The involvement of different organs in the course of the migratory routes of larvae from oral penetration on was specified, pointing out the possible mechanisms of larval access to the fibromuscular layer through kidney, rectum, and vascular emboli. Together with Conejo and Morera (1988), we share the same hypothesis of $\mathrm{L}_{3}$ elimination as an accidental process in which random location near excretory ducts enables larval elimination through mucous secretion after mechanical rupture of the granulomas or perilarval amebocyte reactions. This fact might explain the low recovery of larvae from the mollusk's body, both in natural (Rambo et al. 1997) and experimental infection (Bonetti \& Graeff-Teixeira 1998) since elimination is apparently independent of larval maturation and possibly depends on mechanic and accidental processes. It is relevant to remember that $\mathrm{L}_{3}$ are inactive, or immobile inside the mollusks, unable to achieve active elimination. Indeed, we have observed that some granulomas containing $\mathrm{L}_{3}$ were transported or formed close to excretory ducts, compressing their epithelium (Fig. 6) and occasionally provoking partial epithelial disruption (Figs 7,8 ), thus

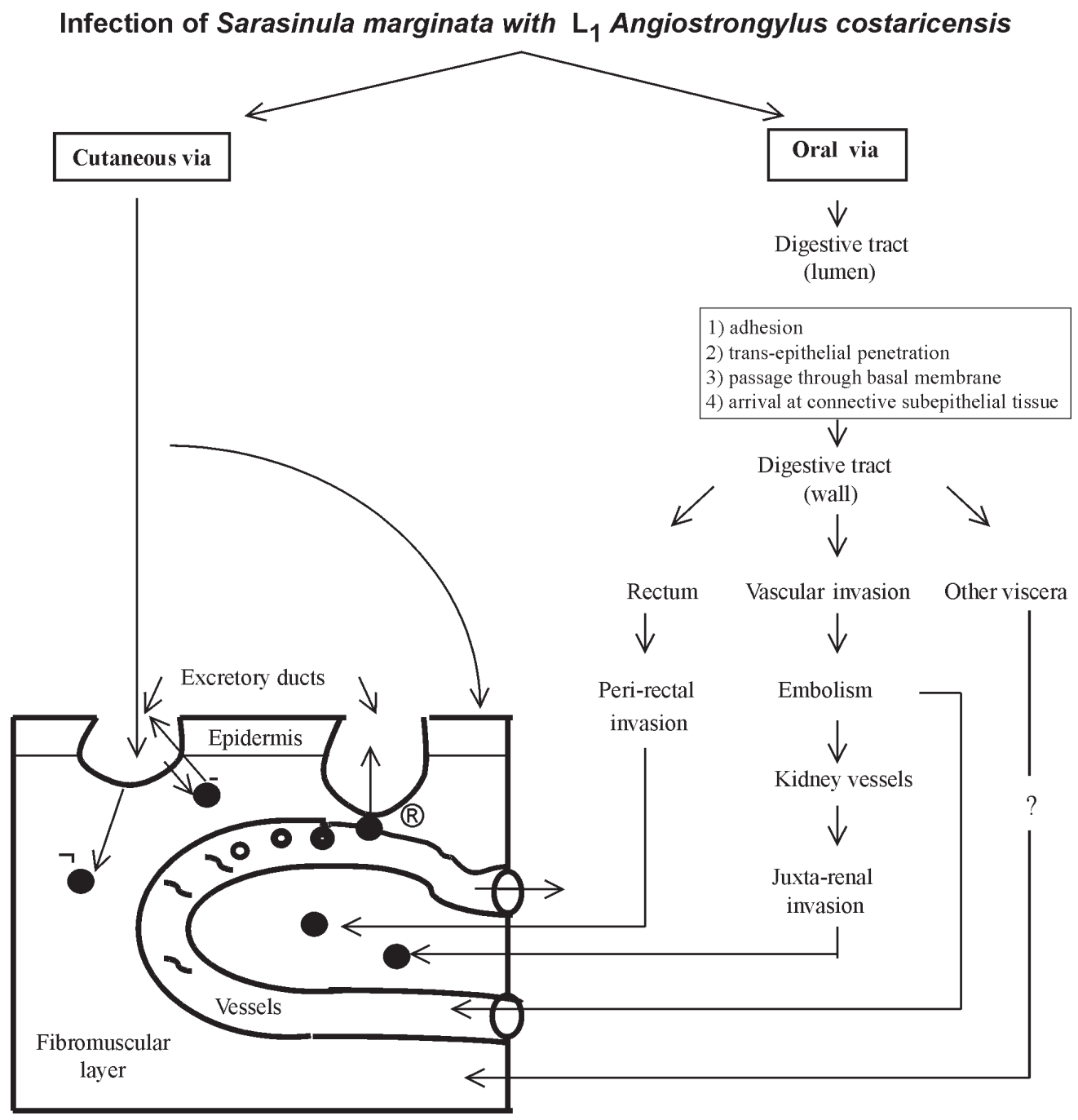

ح : larvae $\left(\mathrm{L}_{1}\right)$; 0 O : pre-granuloma; $\mathrm{O}$ granuloma, $\mathbf{O}$ : subtegumental vessels, $\neg$ : larvae permanently lodged in the libro-

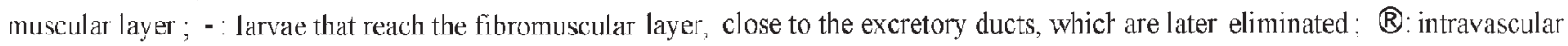
larvae that induce granulomas, which could, after migration, be trapped near excrelory ducls, been further eliminated

Fig. 13: migration model of Angiostrongylus costaricensis in the intermediate host (Sarasinula marginata) 
facilitating $\mathrm{L}_{3}$ elimination. Lie and Heyneman (1976) similarly suggested that parasite encapsulation in the mollusk subepithelial fibromuscular layer resulted in occasional sporocyst elimination in the Echinostoma lindoense-Biomphalaria glabrata model. As amebocyte capsules became thicker, the strata close to the mollusk epithelium became thinner and turgid so that rupture of the epithelium allowed encapsulated sporocyst evasion through it. Richards and Merrit (1967) observed that such capsules persisted for long periods of time and viable Angiostrongylus cantonensis $\mathrm{L}_{3}$ were recovered from Biomphalaria up to 12 months post-infection.

The larvae possibly acquired by cutaneous infection can hypothetically follow three different directions: (1) be kept arrested by the granulomatous reaction in the fibromuscular layer; (2) be lodged close to ducts, and (3) invade vessels, as is the case for larvae acquired by oral infection, being further transported intravascularly and trapped by granulomatous reactions adjacent to excretory ducts, from where they can be possibly eliminated.

The current work also shows that the rectum was highly infected by the parasites. Except for the kidney (Mendonça et al. 1999) and rectum, few larvae were found within other viscera (Table). These two organs seem to be the preferential route for larvae that have orally penetrated to finally reach the fibromuscular layer. They were more intensely parasitized when compared to other organs in all slugs. The access of larvae to the fibromuscular layer was facilitated by the anatomical insertion of the kidney and rectum within it. In fact, larvae surrounding the rectum were found both in the coeloma and in the peripheral portion of the fibromuscular layer (Fig. 4). On the other hand, larvae, which penetrated the fibromuscular layer through the kidney, appeared to have been trapped as emboli in peripheral renal vessels, which are inside this layer (Fig. 3). These results agree with observations made by Richards and Merrit (1967) and Harris and Cheng (1975) in the $A$. cantonensis $-B$. glabrata model, where the importance of kidney and rectum as the main migration routes to the fibromuscular layer was emphasized. However, it was not possible to identify the final habitat of the larvae that reached the fibromuscular layer through those two mentioned organs; it is likely that they are retained in their surroundings. There is a remote possibility that the larvae, after leaving kidney and rectum, could also penetrate the vessels and/or migrate out of the vessels to the epidermal surface for further elimination.

Frequent findings of larvae within the vessels (Figs 1, 2,3 ) are indicative that larvae circulate until they are arrested inside the fibromuscular layer vessels by amebocytic reactions (Figs 8, 9, 10,11,12). According to these data, it is possible that larvae are arrested by the cellular reaction in the viscera (ectopic localization), not being eliminated through mollusk mucus. These larvae are epidemiologically important for life cycle maintenance in nature since they continue to develop even after being arrested and their elimination occurs after digestion in the vertebrate host's stomach. The location of larvae inside the vessels indicates that Angiostrongylid larvae prefer the intravascular habitat in the invertebrate host (Mendonça et al. 1999), or that they only form a passive embolus, since some larvae were detected in the heart and pericardiac cavity (Fig. 5).

Most of the granulomas were well fixed to the endothelium by thin cellular septa formed by fibroblastlike cells from the amebocytic reaction (Fig. 10), leading to the formation of a "marginal sinus" causing thickening of vascular walls (Fig. 11) and dilatation of meta-arterial-like vessels (with segmented sphincters) (Fig. 12).

Our study allows us to conclude that larval access to the fibromuscular layer via the oral route occurs by two different manners: (a) through kidney and rectum, and (b) by vascular emboli. Only those larvae that reached the fibromuscular layer through emboli, contributed to the parasite cycle, eliminating $L_{3}$ Such elimination might depend on intravascular granulomas near the ducts. Larvae that prenetrate through the skin also seem to depend on granulomas located close to the excretory ducts of the mucous cells to be eliminated.

\section{ACKNOWLEDGEMENTS}

To AL de Amorim, FF Cruz, and ID Pedro (Department of Pathology, Instituto Oswaldo Cruz) for technical assistance.

\section{REFERENCES}

Bonetti VCBDO, Graeff-Teixeira C 1998. Angiostrongylus costaricensis and the intermediate hosts: observations on elimination of $\mathrm{L}_{3}$ in the mucus and inoculation of $\mathrm{L}_{1}$ through the tegument of mollucs. Rev Soc Bras Med Trop 31: 289-294.

Carson FL, Martin JH, Lynn JA 1973. Formalin fixation for electron microscopy: a re-evaluation. Am J Clin Pathol 59: 365-375.

Conejo ME, Morera P 1988. Influencia de la edad de los veronicelideos en la infección con Angiostrongylus costaricensis. Rev Biol Trop 36: 519-526.

Harris KR, Cheng TC 1975. The encapsulation process in Biomphalaria glabrata experimentally infected with the metastrongylid Angiostrongylus cantonensis: light microscopy. Int J Parasitol 5: 521-528.

Lennert K 1978. Malignant Lymphomas other than Hodgkin's Disease, Springer-Verlag, Berlin, 833 pp.

Lie KJ, Heyneman D 1976. Studies on resistance in snails. 3. Tissue reactions to Echinostoma lindoense sporocysts in sensitized and resensitized Biomphalaria glabrata snails. J Parasitol 62: 51-58.

Mendonça CLGF, Carvalho OS, Mota EM, Pelajo-Machado M, Caputo LFG, Lenzi HL 1999. Penetration sites of and migratory routes of Angiostrongylus costaricensis in the experimental intermediate host (Sarasinula marginata). Mem Inst Oswaldo Cruz 94: 549-556.

Morera P, Céspedes R 1971a. Angiostrongylus costaricensis n. sp. (Nematoda: Metastrongyloidea), a new lungworm occurring in man in Costa Rica. Rev Biol Trop 18: 173-185.

Morera P, Céspedes R 1971b. Angiostrongilosis abdominal. Una nueva parasitosis humana. Acta Med Costarricense 14: 159-173.

Rambo RP, Agostini AA, Graeff-Teixeira C 1997. Abdominal angiostrongylosis in Southern Brazil - Prevalence and parasitic burden in mollusc intermediate host from eighteen endemic foci. Mem Ins Oswaldo Cruz 92: 9-14.

Richards CS, Merritt JW 1967. Studies on Angiostrongylus cantonensis in molluscan intermediate hosts. J Parasitol 53: 382-388.

Thiengo SC 1996. Mode of infection of Sarasinula marginata (Mollusca) with larvae of Angiostrongylus costaricensis (Nematoda). Mem Inst Oswaldo Cruz 97: 277-278. 